\section{Estudo \\ CoDebate}

em Testão

Planejamento
Revista Estudo \& Debate, Lajeado, v. 27, n. 2, 2020. ISSN 1983-036X

DOI: http://dx.doi.org/10.22410/issn.1983-036X.v27i2a2020.2305

\title{
UM OLHAR SOBRE A INTERNET DAS COISAS EM CIDADES INTELIGENTES: UMA PESQUISA BIBLIOMÉTRICA DOS ANOS DE 1999 A 2019
}

\author{
Alisson Panazzolo ${ }^{1}$, Luís Fernando Moreira ${ }^{2}$, Joel Tshibamba Mukendi ${ }^{3}$
}

\begin{abstract}
Resumo: $\mathrm{O}$ interesse sobre cidades inteligentes surge através do crescimento populacional, responsável pela saturaçáo dos transportes públicos e vias de comunicabilidade, bem como, por aumentar a demanda de provisōes de água e energia. Este estudo teve por objetivo analisar a evolução da produção científica a respeito das temáticas "Cidades inteligentes" e "Internet das coisas". Com base na Lei de Lotka (produtividade científica de autores), Lei de Bradford (produtividade de periódicos) e a Lei de Zipf (frequência de palavras), buscou-se identificar os países que mais publicaram a partir de 1999 até junho de 2019. A pesquisa foi desenvolvida por meio de um estudo bibliométrico na base Web of Science, tendo como critério de análise os trabalhos mais citados, os principais autores (relevantes), as palavras chave, e os países que se destacaram em investigar sobre o tema. Identificou-se um aumento na produção científica na base sobre a temática proposta, os artigos pesquisados demonstraram que o conceito "cidade inteligente" é alvo de críticas por não possuir sentido claro e definido, porém, fica evidente sua relevância dada a devotada busca das cidades a fim de se tornarem "mais inteligentes", com propósitos de sustentabilidade em suas dimensões econômica, social e ambiental, através do incremento da internet das coisas.
\end{abstract}

Palavras-chave: Sustentabilidade. Tecnologia da Informação e Comunicação. Estudo bibliométrico.

\section{LOOK AT THE INTERNET OF THINGS IN INTELLIGENT CITIES: A BIBLIOMETRIC RESEARCH FOR THE YEARS FROM 1999 TO 2019}

Abstract: Interest in smart cities arises through population growth, which is responsible for the saturation of
public transport and communicability, as well as for increasing the demand for water and energy supplies.
The objective of this study was to analyze the evolution of scientific production regarding the themes "Smart
Cities" and "Internet of Things". Based on the Law of Lotka (author's scientific productivity), Bradford's Law

1 Graduando em Administração pela Universidade de Caxias do Sul, bolsista voluntário de Iniciação Científica no grupo Teoria Social das Organizaçóes.

2 Graduado em Engenharia de Produção pela Faculdade Anhanguera, Mestrando em Administração pela Universidade de Caxias do Sul, bolsista de pesquisa no grupo Teoria Social das Organizaçôes.

3 Graduado em Direito Econômico e Social pela Universidade Protestante do Congo e Administração pela Universidade de Caxias do Sul, Mestrando em Administração pela Universidade de Caxias do Sul. 
(productivity of periodicals) and the Law of Zipf (frequency of words), we tried to identify the countries that published the most from 1999 until June 2019. The research was developed by means of a bibliometric study in the Web of Science database, having the most cited works, the main authors (relevant), the keywords, and the countries that stood out in investigating the theme as the criterion of analysis. It was identified an increase in the scientific production in the base on the proposed theme, the articles researched demonstrated that the concept "intelligent city" is criticized for not having clear and definite sense, however, its relevance is evident given the devoted search of the cities in order to become "smarter", for purposes of sustainability in its economic, social and environmental dimensions, by increasing the internet of things.

Keywords: Sustainability. Information and Communication Technology. Bibliometric Study.

\section{Introduçáo}

As cidades inteligentes ou smart cities surgiram a partir da necessidade da busca por novas formas de conciliar o aumento populacional e a expansão da área urbana. Este movimento intensifica estudos sobre os meios de lidar com o crescimento, a fim de gerar um impacto mínimo ao meio ambiente. É abordado para tal, o estilo de vida dos cidadãos, da governança e da utilização das tecnologias de informação e comunicação (TIC's) presentes nas cidades (SILVA; KHAN; HAN, 2018). Harrison et al. (2010) descreve as smart cities como áreas urbanas que exploram seus dados operacionais, decorrente de acontecimentos e serviços prestados pela cidade. Para a otimização dos mesmos, estes dados seriam classificados em três categorias: instrumentado, interconectado e inteligente.

A internet das coisas aplicada em um contexto urbano, responde a uma multiplicidade de anseios governamentais em usar as TICs para auxiliar na gestâo de assuntos públicos, tornando possível, assim, automatizar vários serviços de uma cidade. A chamada Urban IoT trata da utilização de objetos inteligentes em um contexto urbano de maneira a proporcionar serviços de apoio a administração pública das cidades, as empresas e os cidadãos (ZANELLA et al., 2014). Em vista disso, nos últimos anos, foi observado um esforço significativo de pesquisa e desenvolvimento realizado em várias partes do mundo para se criar sistemas computacionais que ajudem a enfrentar os grandes problemas das cidades causados, essencialmente, pelo aumento populacional (HERNANDES et al., 2017).

No cenário brasileiro, o Banco Nacional de Desenvolvimento (BNDES) realizou um estudo a respeito da implementação do IOT no Brasil em 2017. Esse relatório apontou que as administrações governamentais precisam seguir distintas açóes para estimular a inovação. No entanto, vêm realçando o apoio à formação do ecossistema e a diminuição do risco de inovação. Portanto, observa-se que na incitação à inovação, a maior parte dos países atua pelos investimentos diretos, preparação de políticas públicas, concepção de clusters, programas de suporte a pequenas e médias empresas, além de apoio à startups e à demanda. Tais atos incidem em acordos com o setor público e estímulos fiscais, originam oportunidades de suporte e mentora a promoçẫo de cultivo ao empreendedorismo (BNDES, 2017).

Diante da relevância desses termos, o objetivo deste artigo consistiu em analisar a evolução da produção científica a respeito das temáticas "Cidades inteligentes" e "Internet das coisas", principalmente com base na Lei de Lotka (produtividade científica de autores), a Lei de Bradford (produtividade de periódicos) e a Lei de Zipf (frequência de palavras). Ainda buscou identificar os países que mais publicaram a partir de 1999 até junho de 
2019 (LOTKA, 1926; BRADFORD, 1934; ZIPF, 1949). Assim, a estrutura do artigo é composta pela introdução (seção 1), a seguir o referencial teórico que embasa a pesquisa realizada (seção 2), os procedimentos metodológicos escolhidos e utilizados (seção 3), a análise dos resultados (seção 4), e, por fim, as considerações finais (seção 5).

\section{Referencial teórico}

\subsection{Percepção teórica das Cidades Inteligentes}

O crescimento populacional de uma cidade resulta em uma procura na provisão de água, de energia, na saturação dos transportes públicos e vias de comunicabilidade. Também, traz dificuldades a respeito do acesso a serviços de saúde, educação e segurança, caso não seja feito nada pelas entidades competentes para contrariar essas situaçóes. Em outras palavras, diante do crescimento populacional torna-se necessário melhorar a eficiência da estrutura citadina, assim como a qualidade de vida do cidadão. Nesse sentido, fala-se de cidades inteligentes que tiram proveito das TICs (tecnologias de infraestrutura e comunicação) para transformar ou expandir seus sistemas, sua operação e sua entrega de serviços (CENTRAL POLICY UNIT, 2015).

A Universidade de Viena realizou um estudo dirigido por Giffinger et al. (2007), onde pesquisou 58 cidades europeias de porte médio e criou um modelo popularizado de cidade inteligente que auxilia nas pesquisas relacionadas a cidades inteligentes (DAMERI, 2017). Esse estudo propôs um sistema de classificação segundo o qual as cidades podem ser analisadas e aperfeiçoadas por meio de seis características distintas: economia, pessoas, governança, mobilidade, meio ambiente e qualidade de vida (GIFFINGER et al., 2007). Essas seis dimensóes catalisam os 31 fatores detectados nas cidades avaliadas. A Figura 1 apresenta as dimensóes e os fatores observados.

Figura 1 - Dimensões das cidades inteligentes segundo Giffinger e Gudrun (2010)

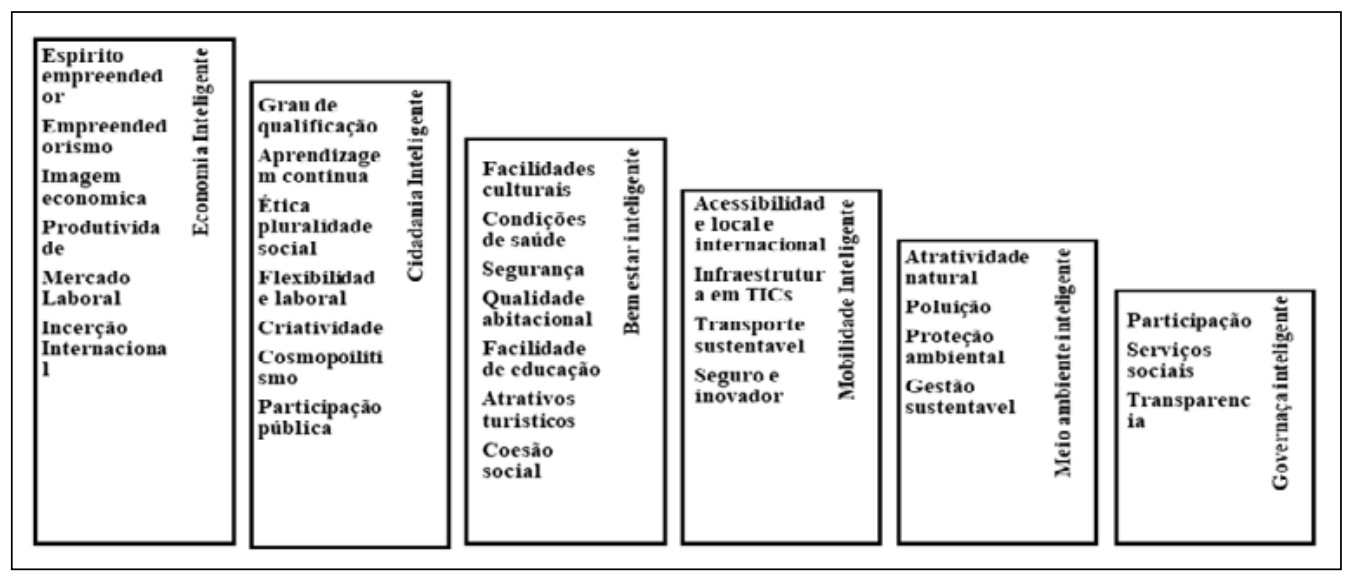

Fonte: Adaptado de Giffinger e Gudrun (2010). 
Conforme mencionado anteriormente, o desafio das cidades inteligentes consiste em acomodar as resoluções tecnológicas com os anseios individuais de seus habitantes, para modificá-las em um valor público que acate aos objetivos econômicos, sociais e ambientais da comunidade (FERRO et al., 2013). Assim, observa-se o interesse dos pesquisadores em estudar a temática, o que proporciona conceituaçóes diversificadas. O Quadro 1 apresenta algumas definiçóes a respeito das cidades inteligentes.

Quadro 1 - Definiçóes para Cidades Inteligentes

\begin{tabular}{|c|c|}
\hline BERRONE et al., (2018) & $\begin{array}{l}\text { Sáo sistemas vivos de indivíduos interagindo e usando } \\
\text { energia, materiais, serviços e financiamento para } \\
\text { catalisar o desenvolvimento econômico e a melhoria da } \\
\text { qualidade de vida. }\end{array}$ \\
\hline $\begin{array}{c}\text { DÍAZ-DÍAZ; MUÑOZ; PÉREZ- } \\
\text { GONZÁLEZ (2017) }\end{array}$ & $\begin{array}{l}\text { A cidade inteligente se beneficia do engajamento de } \\
\text { cidadáos que estão mais bem informados sobre os } \\
\text { serviços públicos e estáo mais comprometidos com seu } \\
\text { gerenciamento. Permitem a criação de um ecossistema } \\
\text { de negócios especializado em produtos e serviços para } \\
\text { cidades inteligentes e, portanto, empregos novos e } \\
\text { altamente qualificados. }\end{array}$ \\
\hline SUJATA et al., (2016) & $\begin{array}{l}\text { As cidades estão cada vez mais conscientes do conceito de } \\
\text { "cidade inteligente" e estão desenvolvendo ativamente } \\
\text { estratégias com o objetivo de se tornarem "inteligentes" } \\
\text { e gerenciar os recursos da cidade de maneira mais } \\
\text { eficiente, ao mesmo tempo em que abordam os desafios } \\
\text { de desenvolvimento e inclusão. }\end{array}$ \\
\hline LUVISI; LORENZINI (2014) & $\begin{array}{l}\text { Uma cidade conectada pela qual as árvores são } \\
\text { conectadas a agências governamentais locais para } \\
\text { sua proteção e gerenciamento pode representar uma } \\
\text { abordagem interessante para um futuro inteligente e } \\
\text { verde. }\end{array}$ \\
\hline $\begin{array}{l}\text { ROSCIA; LONGO; LAZAROIU } \\
\text { (2013) }\end{array}$ & $\begin{array}{l}\text { A Cidade Inteligente refere-se àquele local no contexto } \\
\text { territorial, onde o uso planejado e sensato dos recursos } \\
\text { humanos e naturais, adequadamente manejados e } \\
\text { integrados através das diversas tecnologias de TIC já } \\
\text { disponíveis, permite a criaçáo de um ecossistema que } \\
\text { pode ser usado de recursos e para fornecer sistemas } \\
\text { integrados e mais inteligentes. }\end{array}$ \\
\hline GIFFINGER e GUDRUN (2010) & $\begin{array}{l}\text { O caminho para a cidade se tornar inteligente, demanda } \\
\text { um processo de mudança que envolve três aspectos: uma } \\
\text { nova relação entre stakeholders, a interação desses com } \\
\text { as inovaçóes tecnológicas; e um modelo de governança } \\
\text { adaptativa. }\end{array}$ \\
\hline
\end{tabular}

Fonte: elaborado pelos autores (2019). 


\subsection{Internet das Coisas em Cidades Inteligentes}

A criação da internet contribuiu para o funcionamento das coletividades modernas, com o uso de protocolos de endereçamento tornou-se possível a transmissão entre dispositivos disjuntos fisicamente, partindo de diferentes paradigmas de alcance e do tempo. A possibilidade de utilizar dispositivos como: Identificação por Rádio Frequência (RFID), tags, sensores, atuadores, smartphones, entre outros, conectados à rede em qualquer lugar e hora, iniciou a era da ubiquidade, essencial característica e o pilar fundamental para o surgimento da Internet das Coisas, ou em inglês, Internet of Things (IoT) (TIBURSKI et al., 2015).

A IoT vem sendo utilizada em diferentes áreas, automatização de residências, mensuração avançada, redes inteligentes, iluminação, gestão de tráfego e monitoramento de ambiente. Essas áreas de aplicação estão sendo agregadas ao conceito de cidades inteligentes (JARA et al., 2013). Assim, o número de dispositivos usados para coletar dados das cidades requerem a utilização de tecnologias da IoT, caracterizada pelo alto grau de captura autônoma de dados, transferência de eventos de rede, conectividade e interoperabilidade (CASAGRAS, 2009; SANTANA et al., 2017).

Contudo, segundo Greengard (2015) a IoT refere-se não somente à interconexão entre objetos do mundo real, mas, à contextualização de informaçôes sobre as coisas do mundo real ou sensores. Em outros termos, a IoT se norteia em sensores conectados as coisas que por meio de interfaces eletroeletrônicas de comunicação e controle, possam ser interligadas às redes, inclusive a internet, para que os dados coletados por estes, sejam tratados e correlacionados a outros, e também, com informaçóes de diferentes objetos ou bases de dados existentes, através de aplicativos (apps), para então resultarem em utilidades práticas para os usuários (PERES, 2017).

Nessa perspectiva, Santos (2003) afirma que um sensor se concebe como um dispositivo capaz de captar e eventualmente arquivar informaçóes a respeito de uma determinada variável ou métricas. No entanto, encontram-se diversos tipos de sensores que podem realizar um ou mais tipos de acompanhamento; em todos os casos, adquirindo informaçóes sobre algo dinâmico, que varia ao longo do tempo, registrando os diversos estados ou condiçóes do objeto ou evento de sua atenção.

Assim, o resultado do processamento por uma aplicação ou serviço é uma atuação no ambiente em que estão presentes os objetos e/ou uma atuação sobre eles (CASAGRAS, 2009). 
Figura 2 - Interação entre as redes de comunicação, o mundo físico e o virtual

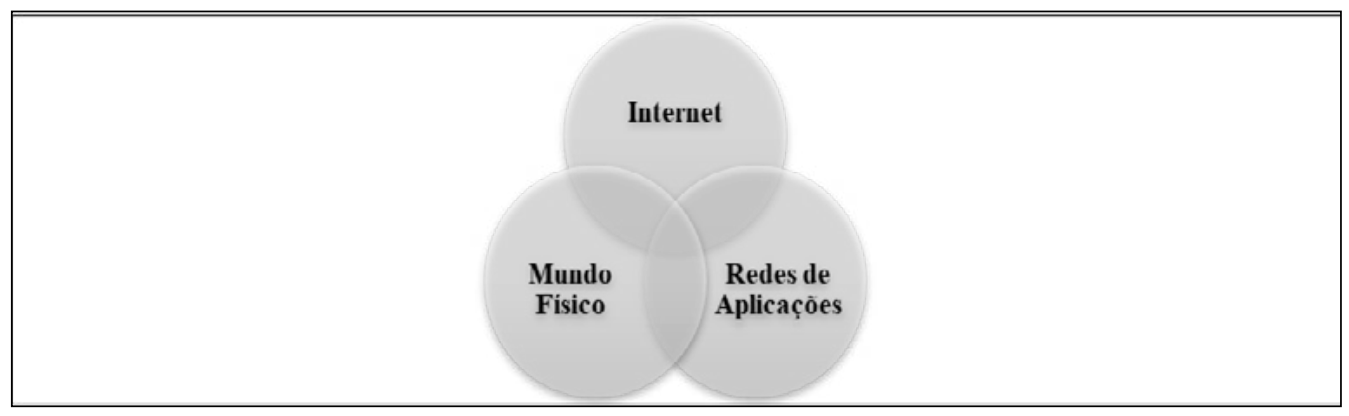

Fonte: Adaptado de CASAGRAS (2009).

Casagras (2009) apresenta três classes de dispositivos para IoT, assim como a Figura 2 apresenta a interação entre as redes de comunicação, o mundo físico e o virtual: i) Objetos puramente passivos com identificação e dados fixos, são objetos presentes na Physical Interface Zone, na parte superior da Figura 2; ii) Objetos dotados de moderado poder computacional e percepçáo de contexto, que por meio de sensores podem gerar mensagens e variar a informação associadas a eles de acordo com o tempo e lugar, representados pelos objetos presentes na Physical Interface Zone, mais ao centro da Figura 2; iii) Objetos que possuem conectividade em rede, sem a intervenção humana, possibilitando a emergência de inteligência nos sistemas de rede, representados pelos objetos presentes na Physical Interface Zone.

Por fim, ao abordar a expressão máquina-a-máquina (M2M), percebe-se que cada sensor monitora uma condição específica, como localização, vibração, movimento e temperatura. Porém, ao falar da IoT, os sensores se conectam entre si por meio de um Wireless Sensor Network (WSN), transmitindo essa informação para um terminal M2M conectado a uma outra rede, para que os sistemas possam entender ou apresentar as informaçóes dos sensores (WANPENG; YU, 2010). A expressão máquina-a-máquina (M2M) refere-se a tecnologias que possibilitam aos sistemas com fio e/ou sem fio a se comunicarem com outros dispositivos que possuem a mesma capacidade, sem intervenção humana direta.

Dessa forma, um dispositivo em um sistema M2M não só coleta dados para seu uso próprio, mas também os compartilha com outros dispositivos, para atingir determinados objetivos (CAO; JIANG; HAN, 2016). A uniformidade da IOT com uma atmosfera inteligente expande as competências dos artefatos inteligentes. $\mathrm{O}$ trabalho em atmosferas inteligentes aperfeiçoados em IOT podem ser classificados nos seguintes campos: Cidades inteligentes, Intelligent Networks, Art Buildings, Intelligent Transport, Intelligent Health e Intelligent Industry (AHMED et al. 2016).

\section{Metodologia}

A abordagem metodológica deste estudo é quantitativa com objetivo descritivo. Desta forma, a técnica usada foi a bibliométrica. Os autores Guedes e Borschiver (2005) conceituam, em sua pesquisa, as principais leis e os princípios bibliométricos, incluindo 
seus objetivos de pesquisa aplicando o conceito cientométrico (ARAÚJO, 2006). Sabese que tal análise consiste em analisar a evolução das publicaçóes científicas, em outras palavras, auxilia a compreender em qual estágio encontra-se a área (TAGUE-SUTCLIFFE, 1992; MACEDO, CASA NOVA, DE ALMEIDA, 2009). Assim, encontram-se as três leis principais da bibliometria: Lei de Bradford (produtividade de periódicos), Lei de Zipf (frequência de palavras) e a Lei de Lotka (produtividade científica de autores).

A pesquisa bibliométrica ocorreu seguindo três etapas nos procedimentos metodológicos. A primeira fase consistiu em escolher a base de consulta Web of Science, tendo em vista que essa base foi escolhida por propiciar ao pesquisador uma estrutura para mapeamento e análise de informaçóes que não requerem grandes manipulaçôes dos dados, e por ter a reputação de ser uma das mais importantes no campo da pesquisa (FINK, 2019; PETTICREW; ROBERTS, 2008). Também, proporciona uma investigação interdisciplinar ao permitir a exploração em profundidade de subcampos especializados dentro de uma disciplina científica (PERES VANTI, 2002; SANTOS, 2003; GARCIA-PEREZ, 2010).

A segunda fase consistiu em delimitar os parâmetros de busca e o período de cobertura da pesquisa. A busca foi realizada apenas pelo campo de pesquisa "title, abstract e keywords", utilizou-se os termos "Smart Cities" and "Internet of Things" com um recorte temporal que abrange de 1999 até junho de 2019. Primeiramente procurou-se por "article" e "conference papers", onde encontrou-se 1.199 documentos em 25 áreas de conhecimento, 43\% são da área de engenharia elétrica e eletrônica; $30,6 \%$ são da área de telecomunicaçóes; $28,52 \%$ da área de sistemas de informação e ciências da computação; 25,43\% da área de métodos e teoria da ciência da computação; e 10,09\% são da área aplicações intermediárias de ciências da computaçáo.

A amostra da pesquisa delimitou-se somente para "article", onde foram apontados 514 trabalhos. Esse total de trabalhos resultou em 55 autores com no mínimo 3 publicaçóes na base, 35 fontes de publicação, 84 instituições relacionadas nas publicações, bem como, salientaram 46 países onde as temáticas estão sendo investigadas. O Quadro 1 apresenta os achados bibliométricos da pesquisa em um contexto mais macro:

Quadro 1 - Dados bibliométricos base Web of Science

\begin{tabular}{|c|c|}
\hline Base de Dados & Web of Science \\
\hline Termos de Busca & "Smart Cities" and "Internet of Things" \\
\hline Campos de Busca & "title, abstract e keywords" \\
\hline Total de Trabalhos Encontrados & 514 \\
\hline Autores & 55 \\
\hline Fontes de Publicação & 35 \\
\hline Instituiçóes & 84 \\
\hline Países & 46 \\
\hline Áreas do Conhecimento & 25 \\
\hline Tipos de Publicação & 2 \\
\hline
\end{tabular}

Fonte: elaborado pelos autores, com base nos dados de Web of Science (2019). 
Para uma visualização e compreensão dos resultados encontrados, foi utilizado o software VOSviewer. Na visão dos autores Van Eck e Waltman (2010) é um software criado para arquitetar e visualizar mapas bibliométricos. Assim, o VOSviewer é usado para construir mapas de autores ou periódicos baseados em dados de co-citaçáo, assim como mapas de palavras-chave baseadas em dados de co-ocorrência. Ainda, oferece também a possibilidade de visualizar os mapas bibliométricos na forma de gráficos (KAPTEIN; HIEMSTRA; KAMPS, 2010). As seçôes em sequência apresentam respectivamente, os resultados retirados da análise com o apoio do software mencionado, consideraçóes finais e possibilidades de futuras pesquisas.

\section{Análise dos dados e discussáo dos resultados}

A análise e discussão dos resultados articulam-se principalmente sobre as principais leis da bibliometria: a Lei de Lotka (produtividade científica de autores), a Lei de Bradford (produtividade de periódicos), e a Lei de Zipf (frequência de palavras) (LOTKA, 1926; BRADFORD, 1934; ZIPF, 1949). Além disso, procurou-se observar quais países possuem maior interesse sobre as temáticas, visualizando assim sua evolução de publicaçóes. As subseçōes a seguir abordam o perfil dos periódicos, publicaçóes por autores, as publicaçóes mais citadas, frequência das palavras-chaves, bem como, os países que mais publicaram durante o período investigado.

\subsection{Perfil dos periódicos}

A partir da Lei de Bradford (1949), foi possível perceber cinco revistas que mais publicaram sobre os termos "Cidades inteligentes" e "Internet das coisas" na base de dados Web of Science. O journal Sensors, em primeiro lugar, aparece com 66 documentos e 409 citaçôes na base. Tem como perfil as áreas do conhecimento da engenharia, eletrônica e elétrica. Seu país de origem é a Suíça e possui um fator de impacto H de 114 de acordo com o Scimago Journal \& Country Rank. Em segundo, o journal Ieee Access, com 53 documentos e 599 citações na base. Tem como áreas do conhecimento, engenharia, ciências da computação e ciências dos materiais. É de origem Americana, e seu fator de impacto H é de 36 de acordo com o Scimago Journal \& Country Rank.

Em terceiro lugar, aparece o journal Future Generation Computer System, com 36 documentos e 349 citaçóes na base. Tem como áreas do conhecimento, redes de computadores, comunicação, hardware e arquitetura de software. Seu país de origem é a Holanda, e seu fator de impacto $\mathrm{H}$ é 85 . O quarto ranqueado foi o journal Ieee Internet of Things, o qual aparece com 36 documentos e 2.014 citações na base. Aborda as áreas do conhecimento de Ciência da Computação, Redes de Computadores e Comunicações, Aplicaçōes em Ciência da Computação, Hardware e Arquitetura, Sistemas de Informação, Processamento de Sinais. O país de origem é o Estados Unidos e seu fator de impacto H é de 31. Em quinto lugar, o journal Ieee Communications Magazine com 10 documentos e 308 citações na base. As áreas do conhecimento abrangem as comunicações, como telecomunicaçóes por ondas de luz, comunicaçôes de dados em alta velocidade e sistemas de comunicação pessoal. O país de origem é Estados Unidos e seu fator de impacto H é de 213. 
Os periódicos com maior número de publicaçóes estão dispostos na Figura 3 de acordo de com a intensidade da cor de cada cluster. Quanto maior a densidade, maior o número de publicaçóes, outro detalhe é que o tamanho de cada cluster também possui significado específico, indicando a quantidade de vezes que tal artigo aparece no grupo de artigos considerados no estudo bibliométrico.

Figura 3 - Os 5 periódicos que mais publicaram sobre "Cidades inteligentes" e "Internet das coisas"

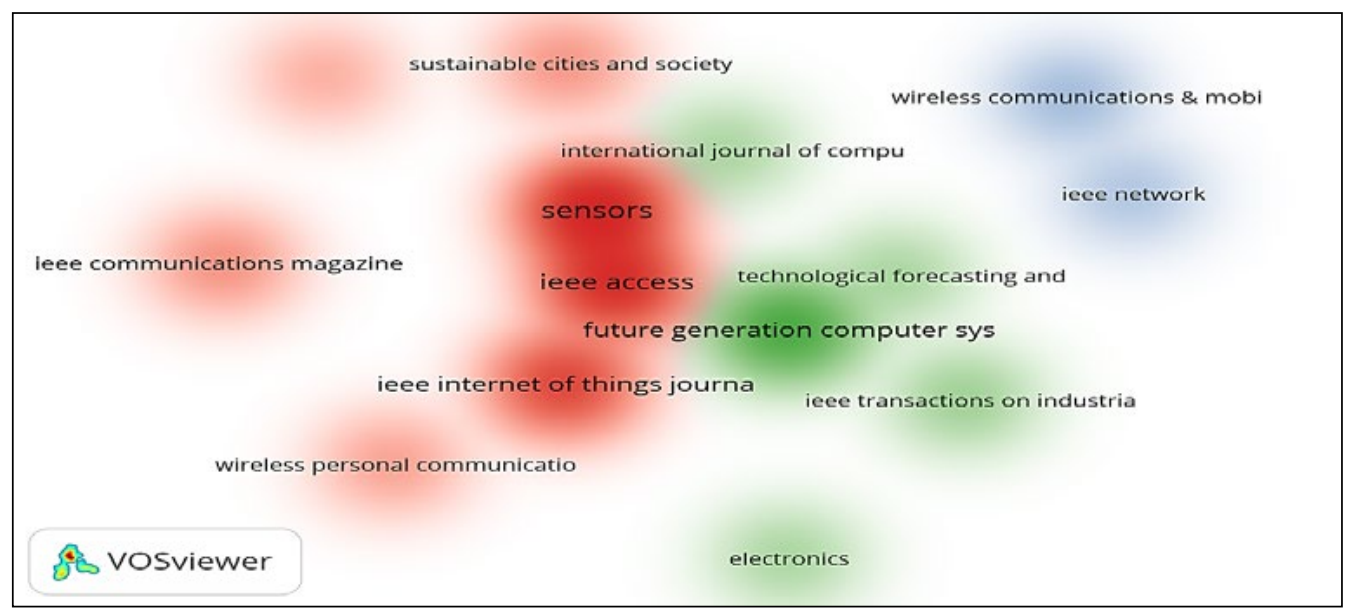

Fonte: Software VOSviewer ${ }^{\circledR}$ (2019).

A descrição da quantidade das publicações demonstra o interesse que os pesquisadores possuem na compreensão dessas temáticas, mas também, da necessidade que os países ou cidades observam em se aprimorarem, isto corrobora com as afirmaçóes de Sujata et al. (2016), que enfatizam que as cidades estáo cada vez mais conscientes do conceito de "cidade inteligente" e estấo desenvolvendo ativamente estratégias com o objetivo de se tornarem "inteligentes", gerenciando os recursos da cidade de maneira mais eficiente, ao mesmo tempo em que aborda desafios de desenvolvimento e inclusão. Para Roscia et al. (2013) uma "Cidade Inteligente" refere-se àquele local no contexto territorial, onde o uso planejado e sensato dos recursos humanos e naturais, adequadamente manejados e integrados através das diversas tecnologias de TIC já disponíveis, permitam a criação de um ecossistema que pode ser usado de recurso para fornecer sistemas integrados e mais inteligentes.

Ademais, essas pesquisas podem se enquadrar na mobilização da área científica a contribuir com os seus respectivos países. Sabendo que, uma cidade inteligente se beneficia do engajamento dos cidadáos que estáo mais informados sobre os serviços públicos e comprometidos com seu gerenciamento, permitem a criação de um ecossistema de negócios especializado em produtos e serviços para cidades inteligentes, e, portanto, empregos novos e altamente qualificados (DÍAZ-DÍAZ; MUÑOZ; PÉREZ-GONZÁLEZ, 2017). 


\subsection{Publicaçáo por Autores}

Seguindo a análise das publicaçóes, é apresentado a seguir os três principais autores encontrados no estudo. Em primeiro lugar foi o autor A. F. Gómez-Skarmeta, que apresentou 8 publicaçóes e foi citado 118 vezes no período analisado. Gómez-Skarmeta é filiado ao departamento de Informática e Sistemas da Universidade de Múrcia, na cidade de Murcia, Espanha. Tem seus estudos voltados para aplicação da IoT para cidades, segurança dos dados das cidades, arquiteturas de redes, iluminação inteligente, big data e energia eficiente. Em segundo, o autor Victor Chang, o qual teve 6 documentos publicados na base e 189 citaçóes. É filiado a Universidade Sino-Britânica fundada em 2006, em Suzhou na província Jiangsu, na república popular da China. O terceiro foi Payam Barnaghi, com 6 documentos e 74 citações na base Web of Science. Atua na área de informática e é filiado a Universidade de Surrey localizada em Guildford, Surrey, Sudeste da Inglaterra, fundada em 9 de setembro de 1966.

A partir dos 514 documentos da busca feita na base de dados através da Lei de Lotka, utilizando o filtro de dois documentos ao mínimo por autor, apresentou-se a seguinte resolução conforme a Figura 4.

Figura 4 - Os 3 autores que mais publicaram artigos na base dados Web of Science

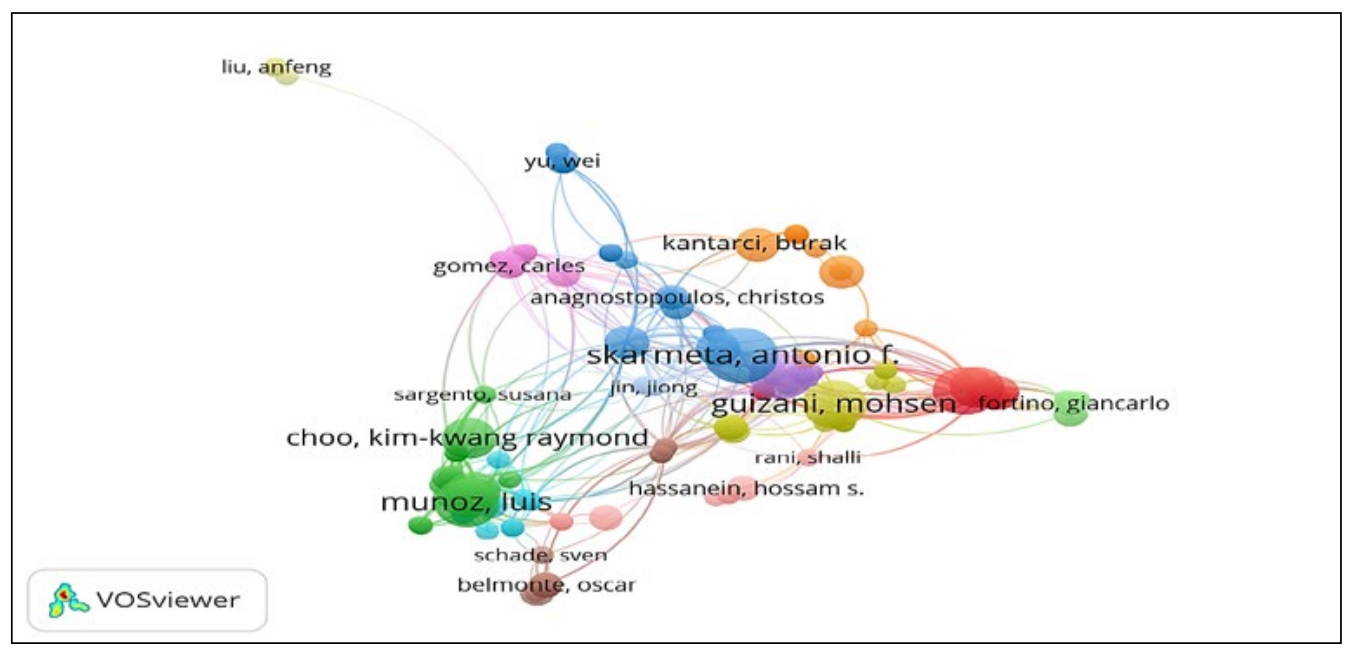

Fonte: Software VOSviewer ${ }^{\oplus}(2019)$.

As análises dos dados para a realização do método Bibliographic Coupling apontou as áreas de centralização de pesquisa onde ocorrem pontos de convergências entre as pesquisas dos três autores: (i) a tecnologia é um dos subsídios estruturantes de uma cidade inteligente; os estudos sobre internet das coisas apresentam maior ênfase, visto que é debatido em profundidade; e, (ii) a pesquisa em IoT é extensa e crescente, e teve máximo foco na hipótese de soluções mais técnicas tais como: unificação dos protocolos de comunicação, segurança dos dados, resiliência do sistema, eficiência energética, armazenamento em larga escala de dados no contexto das cidades e sistemas (PERES, 2017; SANTOS, 2016; ROSCIA, LONGO, LAZAROU, 2013). 
Esses achados ressaltam o interesse que os estudiosos possuem a respeito das temáticas investigadas. Hernandes et al. (2017) enfatizam que nos últimos anos foi observado um esforço significativo de pesquisa e desenvolvimento na aplicaçáo de TICs em cidades, sendo feitas açóes para se criar sistemas computacionais que ajudem a enfrentar os problemas causados essencialmente pelo aumento populacional (BERRONE et al., 2018; HERNANDES et al., 2017).

\subsection{As publicaçóes mais citadas}

Ao contrário da sessão anterior, nesta, será abordado autores os quais tiveram suas obras citadas mais vezes na base de dados objeto deste estudo. Primeiramente com 1.175 citaçóes na base encontra-se o artigo dos autores Zanella, Bui, Castellani, Vangelista e Zorzi (2014) intitulado "Internet of Things for Smart Cities", estudo publicado pelo journal Ieee Internet of Things Jornal. A Figura 5 apresenta o tamanho do cluster referente ao seu número de citaçôes. Em segundo lugar o artigo dos autores Jin, Gubbi, Marusic e Palaniswami (2014) intitulado "An Information Framework for Creating a Smart City Through Internet of Things", teve 352 citaçóes na base e foi publicado no journal Ieee Internet of Things. Em terceiro, o artigo dos autores Perera, Zaslavsky, Christen e Georgakopoulos (2014) intitulado "Sensing as a service model for smart cities supported by Internet of things" teve 298 citações na base e foi publicado no journal Transactions on Emerging Telecommunications Technologies.

Em quarto lugar, o artigo dos autores Lin, Yu, Zhang, Yang, Zhang e Zhao (2017) que se intitula "Survey on Internet of Things: Architecture, Enabling Technologies, Security and Privacy, and Applications", publicado no journal Ieee Internet das coisas Jornal, com 167 citaçóes na base de dados. Por último, o artigo dos autores Gravina, Alinia, Ghasemzadeh e Fortino (2017) intitulado "Multi-sensor fusion in body sensor networks: State-of-the-art and research challenges", publicado no journal Information Fusion, com 159 citaçôes na base Web of Science. As obras presentes na Figura 5, exploram os artigos do estudo relacionando o fator de imediatismo ou citaçóes, que determina o grau de relevância de artigos, cientistas e periódicos científicos, em determinada área do conhecimento. 
Figura 5: Os 5 artigos mais citados na base dados Web of Science

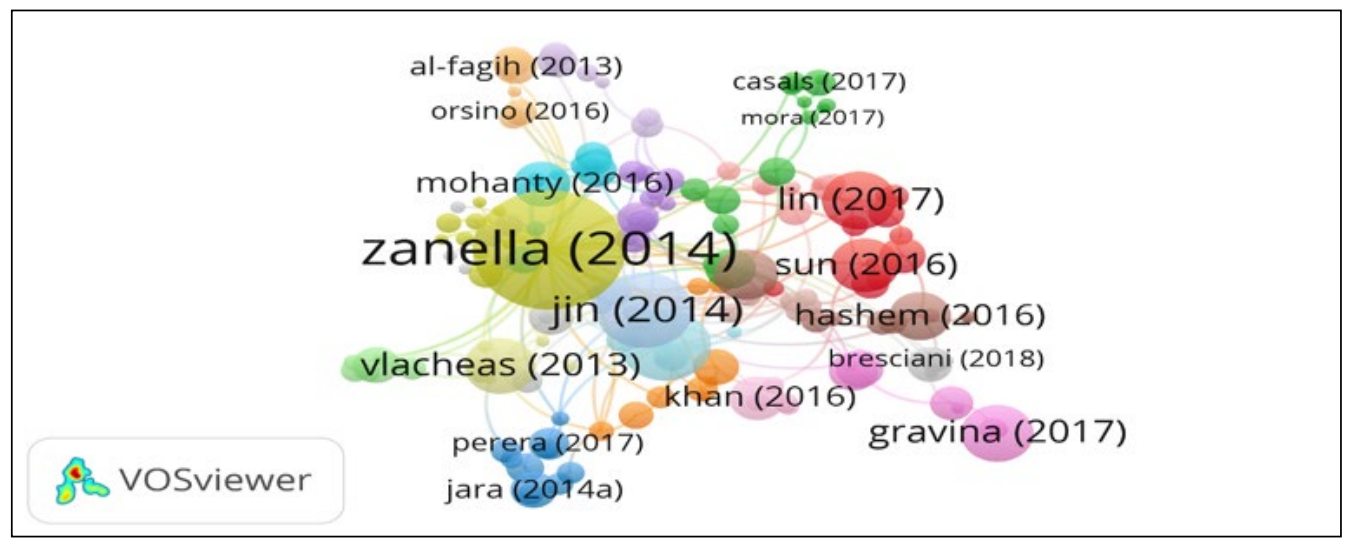

Fonte: Software VOSviewer ${ }^{\circledR}(2019)$

Pode-se constatar que as publicaçóes com maior número de citaçóes, que são mencionadas na temática pesquisada, não incluem os autores que mais publicam sobre o mesmo tema. Os artigos estudados pelos autores são identificados pela análise de citation. Com esta análise, observa-se que os autores com um alto número de publicaçóes, podem não ser aqueles que produzem estudos de máximo impacto e relevância para a apurada área do conhecimento. As abordagens de Zanella et al. (2014), Lin et al. (2017) e Jin et al. (2014) tem uma abordagem que relaciona as Cidades Inteligentes com conceitos mais tecnológicos, como internet e comunicação entre máquinas, representação com prisma de como a computação pode influenciar a cidade inteligente. $\mathrm{O}$ urbanismo inteligente e a cidade inteligente apresentaram meditaçóes a respeito dos problemas e cuidados que insurgem com o tema, assim como o efeito na sociedade civil (SANTANA et al., 2017; WANPENG; YU, 2010).

\subsection{Frequência das Palavras-Chave}

Ao aplicar a Lei de Zipf, encontrou-se uma correlaçáo entre o número de palavras semelhantes e a frequência de seu uso, e conclui-se que existe uma assimetria fundamental na escolha e uso das palavras, e que um pequeno número de palavras é usado mais frequentemente. Primeiramente o termo "Internet of Things" teve uma frequência de 187 menções; em segundo o termo "Smart Cities" aparece com uma frequência de 171 menções; em terceiro o termo "Internet" com 131 mençóes seguido em quarto pelo termo "Things" com 94 mençóes; e, por último, o termo "IoT" 72 mençóes. As palavras Internet of Things e Smart Cities tem a mesma proporção de circunferência corroborando para ligação forte entre os termos. Foi realizada a análise da frequência de palavras dentro do conjunto de palavras-chave informadas nas publicaçóes entre 1999 a 2019, onde se pode registrar as 5 palavras mais ranqueadas. A Figura 6 apresenta a frequência das palavras-chave. 
Figura 6 - As 5 palavras mais citados na base dados Web of Science

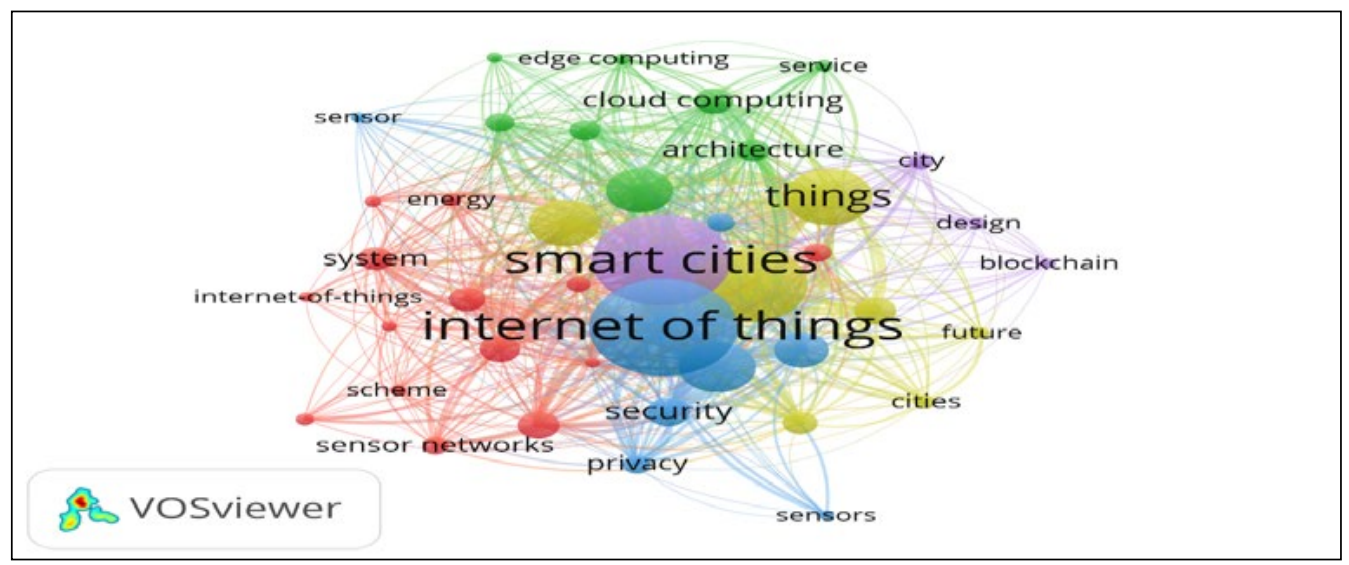

Fonte: Software VOSviewer ${ }^{\circledR}$ (2019).

Nesta análise fica evidente a relação entre a internet das coisas e as cidades inteligentes. Como observado, são estes os termos que aparecem como clusters principais, e em volta dos núcleos centrais os termos relacionadas a temática deste estudo, bem como sensores, sistemas, redes de sensores e computação em nuvem (CAO; JIANG; HAN, 2016; WANPENG; YU, 2010). Nas questōes de aplicação nas cidades, apresentam alguns termos relacionados com o estudo de Giffinger e Gudrun (2010) sobre as dimensóes das cidades inteligentes tais como: segurança e serviço que ficam dentro da dimensão de governança inteligente, a dimensão meio ambiente inteligente aparece pelo termo energia, outros termos como designer, segurança e cidade representam a dimensão bem estar inteligente. As cidades podem ser avaliadas e aprimoradas por meio de seis atributos: economia, pessoas, governança, mobilidade, meio ambiente e qualidade de vida (GIFFINGER; GUDRUN, 2010; GIFFINGER et al., 2007).

\subsection{Países que mais publicaram}

Foi feita uma análise prévia dos países que mais publicam sobre os termos "Cidades inteligentes" e "Internet das coisas" assim mapeando o caminho do conhecimento sobre as pesquisas atuais do referente estudo. Estados Unidos aparece em primeiro lugar com 80 documentos e 820 citaçóes na base; em segundo aparece a Espanha com 73 documentos e 423 citaçóes; em terceiro a China com 61 documentos e 593 citaçóes; na sequência, em quarto a Inglaterra com 35 documentos e 272 citaçôes na base; e, por último, o Canadá, com 26 documentos e 226 citaçóes na base Web of Science. A Figura 7 apresenta esses achados: 
Figura 7 - Os 5 países que mais publicam sobre os termos "Cidades inteligentes" e "Internet das coisas"

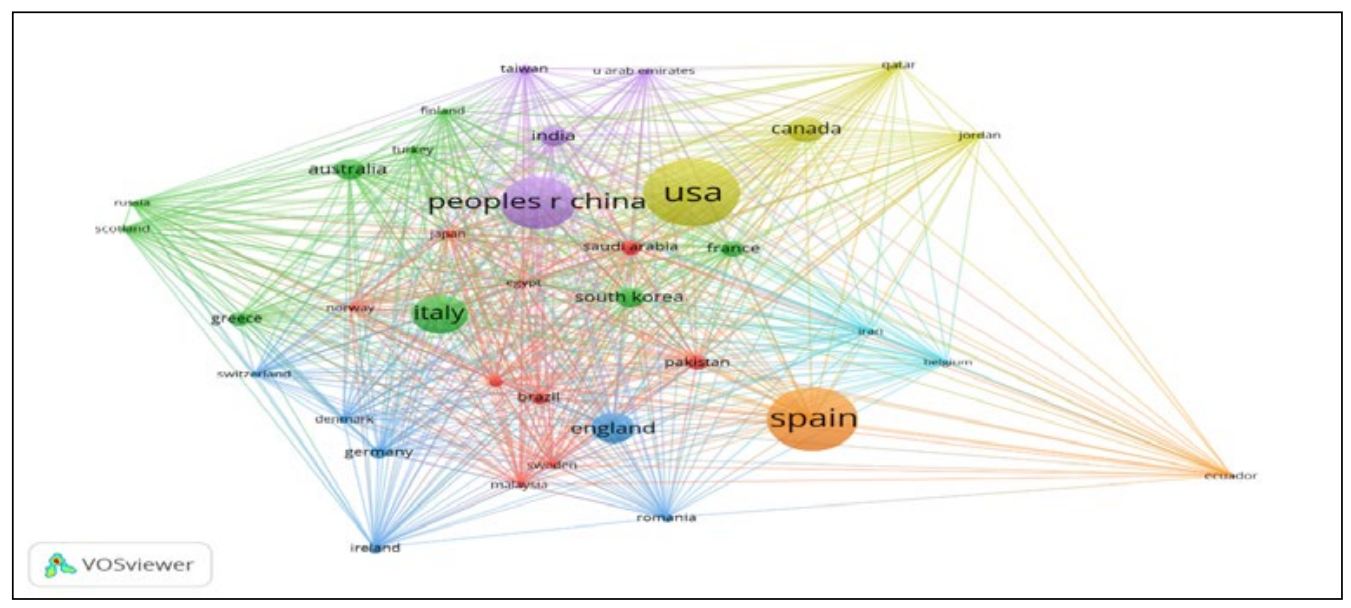

Fonte: Software VOSviewer ${ }^{\varpi}$ (2019).

De acordo com o estudo de Berrone et al. (2018) o continente europeu é onde se encontram as principais cidades inteligentes do mundo que empregam a internet das coisas nas cidades, tendo já projetos consolidados como Barcelona na Espanha, Copenhague na Dinamarca, Murcia na Espanha, Florença na Itália. No geral são 57 cidades. A América do Norte tem o maior números de documentos, mas segundo Berrone et al. (2018) tem 18 cidades consideradas como inteligentes que empregam alguma tecnologia no seu contexto urbano. Os Estados Unidos possuem 14 cidades tais cidades como: Nova York, Miami e Boston. O continente Asiático se destaca com a China com maior número de documentos publicados. O país possui seis cidades: Beijing, Guangzhou, Hong Kong, Shanghai, Shenzhen e Tianjin como modelos de cidades inteligentes das 21 cidades inteligentes do continente Asiático (BERRONE et al., 2018).

Um ponto de convergência entre os três países é sobre o papel do capital humano nas cidades inteligentes, ou seja, qual a relaçáo das cidades inteligentes com as "pessoas inteligentes", no sentido de estarem cientes e com nível de instrução aceitável para operar ou usar as tecnologias IoT em seu auxílio próprio e para com outro indivíduo, a busca do conhecimento e sua disseminaçáo para bem estar social, econômico e ambiental (SANTANA et al., 2017; GIFFINGER; GUDRUN, 2010).

\section{Consideraçóes Finais}

O objetivo desse estudo bibliométrico foi atingido, uma vez que o artigo consistiu em analisar a evolução da produção científica a respeito das temáticas "Cidades inteligentes" e "Internet das coisas". Considerando como base a Lei de Lotka (produtividade científica de autores), apontou-se que o autor A. F. Gómez-Skarmeta apresentou 8 publicaçóes e foi citado 118 vezes no período; já de acordo com a Lei de Bradford (produtividade de periódicos), verificou-se que o artigo dos autores Zanella et al. (2014) intitulado Internet das 
coisas para Cidades inteligentes possui 1175 citações na base e foi publicado no Ieee Internet of Things Jornal; em relação a Lei de Zipf (frequência de palavras), os achados apontaram que a palavra Internet das coisas teve uma frequência de 187 mençôes seguida pela palavra Smart Cities que aparece com uma frequência de 171 mençóes.

Os resultados da pesquisa salientam o crescimento no interesse que os pesquisadores possuem em relação a temática "Cidades inteligentes" e "Internet das coisas", também o interesse que os governos dos países demonstram em tornar as suas cidades inteligentes, utilizando Internet das coisas como base. Observou-se que são os países com economias mais desenvolvidas que mais pesquisam sobre a temática. Nesse sentido, Estados Unidos aparece em primeiro lugar com 80 documentos e 820 citaçóes na base, em segundo a Espanha com 73 documentos e 423 citaçôes; em terceiro a China com 61 documentos e 593 citaçóes; seguida pela Inglaterra em quarto com 35 documentos e 272 citaçóes na base; e, por último, o Canadá com 26 documentos e 226 citações na base Web of Science.

Os autores Zanella et al. (2014), Lin et al. (2017) e Jin et al. (2014) abordaram o conceito da tecnologia e a sua relação com as cidades inteligentes. A Internet das coisas foi apresentada como um desenvolvimento a partir dos desafios existentes do crescimento populacional dos últimos anos, como a dilatação do volume de dados; constituindo-se atualmente em ambientes de inovação tecnológica para elevação da qualidade de vida e da sustentabilidade nas cidades. (AHMED, E. et al. 2016).

As dimensões das cidades inteligentes tais como: economia inteligente, cidadania inteligente, bem-estar inteligente, mobilidade inteligente, meio ambiente inteligente $\mathrm{e}$ governança inteligente são abordadas pelos artigos relacionados neste estudo (GIFFINGER; GUDRUN, 2010; GIFFINGER et al., 2007). Os artigos pesquisados demonstraram que o conceito "cidade inteligente" é alvo de críticas por não possuir sentido claro e definido, ainda que seja evidente a sua relevância dada a devotada busca das cidades a fim de se tornarem "mais inteligentes", seja com propósitos de sustentabilidade em suas dimensōes econômica, social e ambiental; (BERRONE et al., 2018; DÍAZ-DÍAZ; MUÑOZ; PÉREZGONZÁLEZ, 2017; FERRO et al., 2013; GIFFINGER; GUDRUN, 2010).

Por fim, a principal limitação desta pesquisa refere-se ao universo pesquisado e aos parâmetros de busca, visto que foi utilizado uma base de dados como fonte de pesquisa. Sendo assim, sugerem-se estudos futuros que analisem a evolução da produção científica a respeito da sustentabilidade em outras bases, ampliando-se o campo de busca. Além de propor estudos semelhantes relacionados à evoluçáo e relaçóes feitas a respeito de "Cidades inteligentes" e "Internet das coisas" em regiôes economicamente emergentes do mundo, podendo ser feita uma revisão sistemática de literatura utilizando o método de cluster para identificar os principais estudos e fazer os pontos convergentes e divergentes sobre o tema relacionado neste estudo, bem como os métodos de pesquisa aplicados com o tema "Cidades inteligentes" e "Internet das coisas". 


\section{Referências}

AHMED, Ejaz et al. Internet-of-things-based smart environments: state of the art, taxonomy, and open research challenges. IEEE Wireless Communications, v. 23, n. 5, p. $10-16,2016$.

ARAÚJO, Carlos AA. Bibliometria: evolução histórica e questōes atuais. Em questão, v. 12, n. 1, p. 11-32, 2006.

\section{BANCO NACIONAL DE DESENVOLVIMENTO ECONÔMICO E SOCIAL} (BRASIL). BRASIL. Ministério do Planejamento, Desenvolvimento e Gestão. BRASIL. Ministério da Ciência, Tecnologia, Inovações e Comunicaçóes. Produto 1: benchmark de iniciativas e políticas públicas: relatório final. [Rio de Janeiro]: [Banco Nacional de Desenvolvimento Econômico e Social], 2017. 227 p. Disponível em: <https://web.bndes. gov.br/bib/jspui/handle/1408/12496>. Acesso em: 10 dez. 2019.

BERRONE, Pascual et al. IESE cities in motion index 2018. Retrieved, 2018. Disponível em: <https://media.iese.edu/research/pdfs/ST-0471-E.pdf>. Acesso em: 8 Mai. 2018.

BRADFORD, Samuel C. Sources of information on specific subjects. Engineering, v. 137, p. 85-86, 1934.

CAO, Yang; JIANG, Tao; HAN, Zhu. A survey of emerging M2M systems: Context, task, and objective. IEEE Internet of Things Journal, v. 3, n. 6, p. 1246-1258, 2016.

CASAGRAS, E. F. P. Casagras final report: Rfid and the inclusive model for the internet of things. EU FP7 Project CASAGRAS, 2009. Disponível em: <https://docbox.etsi.org/ zArchive/TISPAN/Open/IoT/low\%20resolution/www.rfidglobal.eu\%20CASAGRAS\%20 IoT\%20Final\%20Report\%20low\%20resolution.pdf>. Acesso em: 12 dez. 2018.

CAO, Yang; JIANG, Tao; HAN, Zhu. A survey of emerging M2M systems: Context, task, and objective. IEEE Internet of Things Journal, v. 3, n. 6, p. 1246-1258, 2016.

CENTRAL POLICY UNIT (CPU). The Government of Hong Kong Special Administrative Region. Research Report on Smart City. 2015. Disponível em: <https:// www.pico.gov.hk/doc/en/research_reports/CPU\%20research\%20report\%20-\%20 Smart\%20City(en).pdf>. Acesso em: 23 set. 2019.

DAMERI, Renata Paola et al. Smart city implementation. Progress in IS; Springer: Genoa, Italy, 2017.

DA SILVA MACEDO, Marcelo Alvaro; DE CASTRO CASA NOVA, Silvia Pereira; DE ALMEIDA, Katia. Mapping and bibliometric analysis of the Use of Data Envelopment Analysis (DEA) in accounting and administration in Brazil. CONTABILIDADE

GESTAO E GOVERNANCA, v. 12, n. 3, p. 87-101, 2009. 
DÍAZ-DÍAZ, Raimundo; MUÑOZ, Luis; PÉREZ-GONZÁLEZ, Daniel. Business model analysis of public services operating in the smart city ecosystem: The case of SmartSantander. Future Generation Computer Systems, v. 76, p. 198-214, 2017.

FERRO, E. et al. The role of ict in smart city governance. In: International Conference for E-Democracy and Open Government, Krems, Austria. 2013.

FINK, Arlene. Conducting research literature reviews: From the internet to paper. Sage publications, 2019.

GARCÍA-PÉREZ, Miguel A. Accuracy and completeness of publication and citation records in the Web of Science, PsycINFO, and Google Scholar: A case study for the computation of $h$ indices in Psychology. Journal of the American society for information science and technology, v. 61, n. 10, p. 2070-2085, 2010.

GIFFINGER, Rudolf; PICHLER-MILANOVIĆ, Nataša. Smart cities: Ranking of European medium-sized cities. Centre of Regional Science, Vienna University of Technology, 2007. Disponível em: < http://www.smart-cities.eu/download/smart_cities_ final_report.pdf>. Acesso em: 15, abr. 2019.

GIFFINGER, Rudolf; GUDRUN, Haindlmaier. Smart cities ranking: an effective instrument for the positioning of the cities?. ACE: architecture, city and environment, v. 4, n. 12, p. 7-26, 2010.

GRAVINA, Raffaele et al. Multi-sensor fusion in body sensor networks: State-of-the-art and research challenges. Information Fusion, v. 35, p. 68-80, 2017.

GREENGARD, Samuel. The internet of things. MIT press, 2015.

GUEDES, Vânia LS; BORSCHIVER, Suzana. Bibliometria: uma ferramenta estatística para a gestão da informação e do conhecimento, em sistemas de informação, de comunicação e de avaliação científica e tecnológica. Encontro Nacional de Ciência da Informaçáo, v. 6, n. 1, p. 1-18, 2005.

HARRISON, Colin et al. Foundations for smarter cities. IBM Journal of research and development, v. 54, n. 4, p. 1-16, 2010.

JARA, Antonio J. et al. Mobile discovery: A global service discovery for the internet of things. In: 2013 27th International Conference on Advanced Information Networking and Applications Workshops. IEEE, 2013. p. 1325-1330.

JIN, Jiong et al. An information framework for creating a smart city through internet of things. IEEE Internet of Things journal, v. 1, n. 2, p. 112-121, 2014. 
KAPTEIN, Rianne; HIEMSTRA, Djoerd; KAMPS, Jaap. How different are language models and word clouds?. In: European conference on information retrieval. Springer, Berlin, Heidelberg, 2010. p. 556-568.

LIN, Jie et al. A survey on internet of things: Architecture, enabling technologies, security and privacy, and applications. IEEE Internet of Things Journal, v. 4, n. 5, p. 1125-1142, 2017.

LOTKA, Alfred J. The frequency distribution of scientific productivity. Journal of the Washington academy of sciences, v. 16, n. 12, p. 317-323, 1926.

HERNANDES, Sediane C. L. et al. Serviços de Emergência em Cidades Inteligentes: o Problema de Acionamento de Unidades Móveis. Revista Eletrônica de Sistemas de Informação, v.16, n. 2, p.1-31, 2017.

LUVISI, Andrea; LORENZINI, Giacomo. RFID-plants in the smart city: Applications and outlook for urban green management. Urban forestry \& urban greening, v. 13, n. 4, p. 630-637, 2014.

PERERA, Charith et al. Sensing as a service model for smart cities supported by internet of things. Transactions on emerging telecommunications technologies, v. 25, n. 1, p. $81-93,2014$.

PERES, João Roberto. Manifesto: direitos globais de IoT (livro eletrônico). ed do autor. São Paulo, SP, 2017. Disponível em: <http://www.ntsc-br.com/data/documents/e-BookIoT-MANIFESTO-Edicaolqp.pdf>. Acesso em: 20 dez. 2018.

PERES VANTI, Nadia Aurora. Da bibliometria à webometria: uma exploração conceitual dos mecanismos utilizados para medir o registro da informação e a difusão do conhecimento. Ciência da informaçáo, v. 31, n. 2, p. 152-162, 2002.

PETTICREW, Mark; ROBERTS, Helen. Systematic reviews in the social sciences: A practical guide. John Wiley \& Sons, 2008.

ROSCIA, Mariacristina; LONGO, Michela; LAZAROIU, George Cristian. Smart City by multi-agent systems. In: 2013 International Conference on Renewable Energy

Research and Applications (ICRERA). IEEE, 2013. p. 371-376.

SANTANA, Eduardo Felipe Zambom et al. Software platforms for smart cities: Concepts, requirements, challenges, and a unified reference architecture. ACM Computing Surveys (Csur), v. 50, n. 6, p. 1-37, 2017.

SANTOS, Raimundo Nonato Macedo dos. Indicadores estratégicos em ciência e tecnologia: refletindo a sua prática como dispositivo de inclusão/exclusão.

TransInformaçáo, v. 15, n. SPE, p. 129-140, 2003. 
SILVA, Bhagya Nathali; KHAN, Murad; HAN, Kijun. Towards sustainable smart cities: A review of trends, architectures, components, and open challenges in smart cities.

Sustainable Cities and Society, v. 38, p. 697-713, 2018.

SUJATA, Joshi et al. Developing smart cities: an integrated framework. Procedia

Computer Science, v. 93, p. 902-909, 2016.

TAGUE-SUTCLIFFE, Jean. An introduction to informetrics. Information processing \& management, v. 28, n. 1, p. 1-3, 1992.

TIBURSKI, Ramão Tiago et al. The importance of a standard security architecture for SOA-based iot middleware. IEEE Communications Magazine, v. 53, n. 12, p. 20-26, 2015.

VAN ECK, Nees; WALTMAN, Ludo. Software survey: VOSviewer, a computer program for bibliometric mapping. scientometrics, v. 84, n. 2, p. 523-538, 2010.

WANPENG, F; YU, L. Opportunities, Challenges and Practices of the Internet of Things. 2010. Disponível em: <http://wwwen.zte.com.cn/endata/magazine/ ztetechnologies/2010/no5/articles/201005/t20100510_184418.html>. Acesso em: 03, mai. 2019.

ZANELLA, Andrea et al. Internet of things for smart cities. IEEE Internet of Things journal, v. 1, n. 1, p. 22-32, 2014.

ZIPF, George Kingsley. Human behavior and the principle of least effort Cambridge. MA: Addison-Wesley, 1949. 